University of Nebraska - Lincoln

DigitalCommons@University of Nebraska - Lincoln

October 2005

\title{
Magnetization reversal of Fe films on Ag submicrometer pyramidal islands
}

Chin-Chung Yu

Dept. of Appl. Phys., Nat. Univ. of Kaohsiung, Taiwan

Yeong-Der Yao

Dept. of Appl. Phys., Nat. Univ. of Kaohsiung, Taiwan

Sy_Hwang Liou

University of Nebraska-Lincoln, sliou@unl.edu

Follow this and additional works at: https://digitalcommons.unl.edu/physicsliou

Part of the Physics Commons

Yu, Chin-Chung; Yao, Yeong-Der; and Liou, Sy_Hwang, "Magnetization reversal of Fe films on Ag submicrometer pyramidal islands" (2005). Si-Hwang Liou Publications. 60.

https://digitalcommons.unl.edu/physicsliou/60

This Article is brought to you for free and open access by the Research Papers in Physics and Astronomy at DigitalCommons@University of Nebraska - Lincoln. It has been accepted for inclusion in Si-Hwang Liou Publications by an authorized administrator of DigitalCommons@University of Nebraska - Lincoln. 


\title{
Magnetization Reversal of Fe Films on Ag Submicrometer Pyramidal Islands
}

\author{
Chin-Chung $\mathrm{Yu}^{1}$, Yeong-Der Yao ${ }^{2}$, and Yung Liou ${ }^{2}$ \\ ${ }^{1}$ Department of Applied Physics, National University of Kaohsiung, Kaohsiung 811, Taiwan, R.O.C. \\ ${ }^{2}$ Institute of Physics, Academia Sinica, Taipei 115, Taiwan, R.O.C.
}

\begin{abstract}
Ag submicrometer pyramidal islands with a (100) truncated top and four (111) sidewalls were successfully fabricated on Si(100) substrates at a deposition temperature of $500{ }^{\circ} \mathrm{C}$. The magnetization reversal of $30 \mathrm{~nm}$ thick Fe films grown on Ag pyramidal islands was studied by magnetic force microscopy. The magnetization reversal of Fe films was first started at the sidewalls of pyramidal islands. Then, the formation and propagation of wall-like structures were observed under a higher external magnetic field, namely 95 Oe. The magnetic domain images indicated that the Fe films grown on the sidewalls of pyramidal islands and the strength of external field played important roles on the magnetization reversal of Fe films.
\end{abstract}

Index Terms-Iron films, magnetization reversal, silver pyramidal islands.

\section{INTRODUCTION}

$\mathbf{I}$ T IS well known that the film thickness, shape, and surface/interface roughness are the most important factors on the magnetic behavior of materials than other factors, such as crystalline structure, while reducing the size or dimension of the magnetic structure [1]-[4]. Especially for the ultra-thin film, superlattice, and nanostructured magneto-devices, these parameters will strongly influence the magnetic anisotropy, coercivity, magnetic domain, and magneto-transport properties of the magnetic materials. For small magneto-devices, surface/interface effects are more important than others. In this paper, a $30 \mathrm{~nm}$ thick Fe film was grown on the $\mathrm{Si}(100)$ substrates with a $40 \mathrm{~nm}$ thick Ag buffer layer. For Ag atoms deposited on Si substrates, a Stranski-Krastanov growth mode was found previously [5], [6]. Here, the molecular-beam epitaxial technique was employed to grow the Ag layer on $\mathrm{Si}(100)$ substrates. Unlike the previous study in the system of Fe grown on Ag islands [7]. The Ag layer formed submicrometer pyramidal islands with truncated top. The magnetization reversal of Fe films under the influence of Ag pyramidal islands will be discussed.

\section{PREPARATION AND Characterization of Ag PYRAMIDAL ISLANDS}

In this study, the Ag submicrometer pyramidal islands were grown on highly doped p-type $\mathrm{Si}(100)$ substrates. The $\mathrm{Si}(100)$ substrates were first cleaned by RCA method, which was first developed by the RCA laboratories, Princeton, NJ, in 1970 [8], [9], and then heated to $650{ }^{\circ} \mathrm{C}$ for half an hour under ultra-high vacuum environment. The Ag layer was then deposited on the $\mathrm{Si}(100)$ substrate at $500{ }^{\circ} \mathrm{C}$ by a Kundsen cell with a deposition rate of around $0.05 \AA / \mathrm{s}$ in an Eiko EL-10A molecular-beam epitaxy (MBE) deposition system. The same as previous reports [10], Ag films formed face-centered cubic (fcc) (100) structure

Digital Object Identifier 10.1109/TMAG.2005.854708

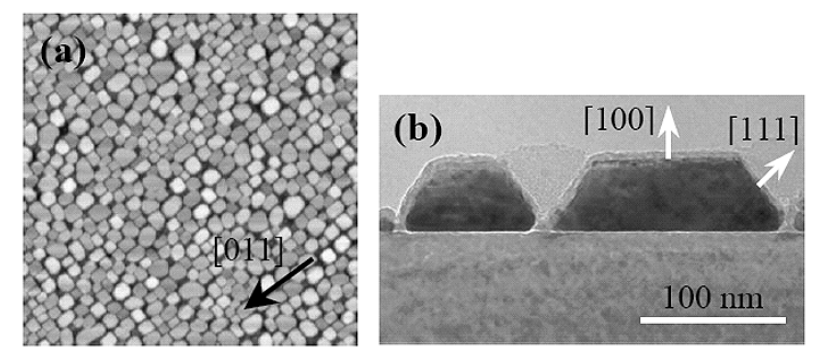

Fig. 1. (a) $5 \times 5 \mu \mathrm{m}$ AFM scan and (b) TEM picture of Ag pyramidal islands.

on $\operatorname{Si}(100)$ substrates with a $4: 3$ coincidence growth. From reflection high-energy electron diffraction (RHEED) [10], we determined the crystal relationship between $\mathrm{Ag}$ and $\mathrm{Si}$, that is, $\mathrm{Si}(100)[011] \| \operatorname{Ag}(100)[011]$. The investigation of atomic force microscopy (AFM) showed that the Ag films formed square-like islands with island edges parallel to $\mathrm{Si}\{011\}$ in-plane directions, as shown in Fig. 1(a) for $40 \mathrm{~nm}$ thick Ag films. The average lateral dimension and height of $\mathrm{Ag}$ islands were of around 150 and $50 \mathrm{~nm}$, respectively. The investigation of transmission electron microscopy (TEM) also suggested the same crystal structure as mentioned above. Furthermore, it indicated that the sidewalls of $\mathrm{Ag}$ pyramidal islands met at an angle of $54^{\circ}$ to the $\mathrm{Ag}(100)$ truncated top as depicted in Fig. 1(b). It suggested that the sidewalls of Ag islands were just fcc $\{111\}$ facet structures. From the above investigations, the Ag films formed submicrometer pyramidal islands with a (100) truncated top and four (111) sidewalls on $\mathrm{Si}(100)$ substrates. More detailed investigation of RHEED and AFM can be found in [10].

\section{RESULTS AND DISCUSSION}

A $30 \mathrm{~nm}$ thick Fe film was then deposited on the Ag pyramidal islands at $100^{\circ} \mathrm{C}$ to avoid the interdiffusion between $\mathrm{Ag}$ and Fe atoms. Fig. 3(a) showed the surface morphology of $\mathrm{Fe}$ films. As our expectation, the morphology of Fe films followed the shape of underlying Ag pyramidal islands. From the previous investigation [10], a strongly bcc(100) texture was observed by RHEED. It also showed that the Fe lattice, that grown 


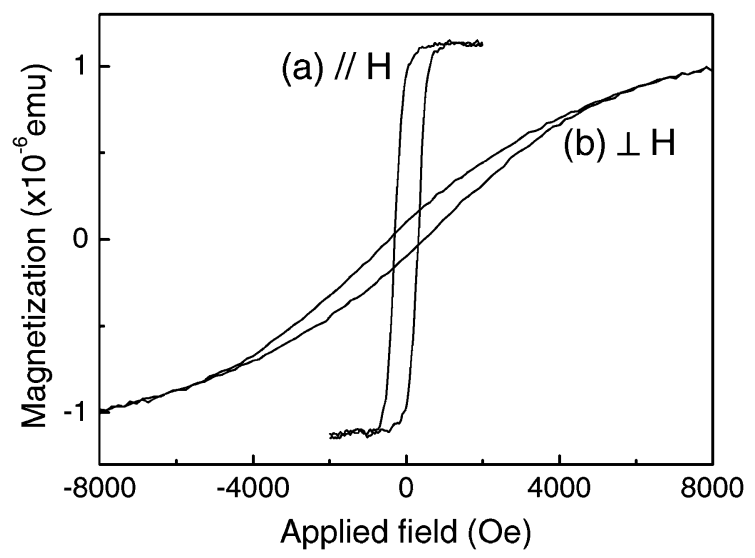

Fig. 2. Hysteresis loops of $30 \mathrm{~nm}$ thick Fe films grown on $40 \mathrm{~nm}$ thick $\mathrm{Ag}$ pyramidal islands. (a) and (b) are for the external magnetic field parallel and perpendicular to film plane, respectively.

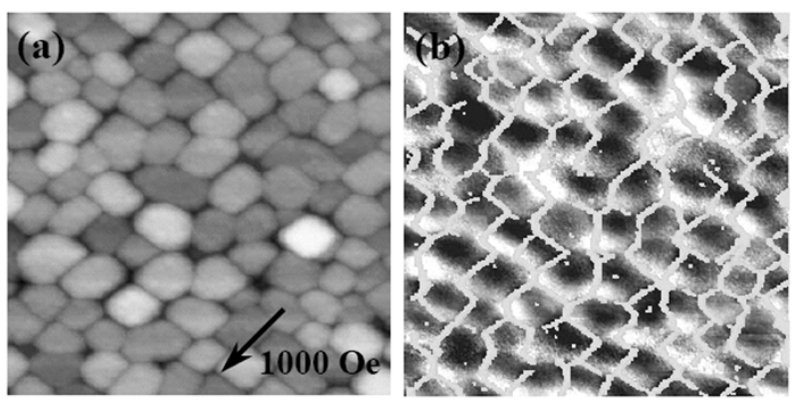

Fig. 3. $2 \times 2 \mu \mathrm{m}$ AFM (a) and MFM (b) images of Fe films. Images were taken under a 1000 Oe external magnetic field with a direction parallel to $\mathrm{Ag}[011]$ as indicated by the arrow in (a).

on the top surface of $\mathrm{Ag}$ pyramidal islands, rotates an angle of $45^{\circ}$ to match the underlying $\mathrm{Ag}$ lattice as reported by other groups [11]. The hysteresis loops of Fe films were measured by a vibration sample magnetometer. The coercive field of the $\mathrm{Fe}$ film grown on $40 \mathrm{~nm}$ thick $\mathrm{Ag}$ pyramidal islands was 309 Oe and 455 Oe for the external field parallel, Fig. 2(a), and perpendicular, Fig. 2(b), to the film plane, respectively. It shows that the Fe film grown on Ag pyramidal islands has perpendicular hysteresis behavior. Note that Fe films were almost magnetically isotropic in the film plane [10].

The magnetic force microscopy (MFM) was taken under different external magnetic fields by a Veeco DI 3100 scanning probe microscope under lift-mode operation. The magnetic field was applied along $\mathrm{Ag}[011]$ or [0-1-1] in-plane direction, as indicated by a black arrow in the down-right corner of AFM pictures.

Figs. 3(a) and (b) were the $2 \times 2 \mu \mathrm{m} \mathrm{AFM} \mathrm{and} \mathrm{MFM} \mathrm{im-}$ ages of $\mathrm{Fe}$ films grown on $\mathrm{Ag}$ pyramidal islands under an external field with strength of 1000 Oe that applied along $\mathrm{Ag}$ [011] in-plane direction. The gray lines drawn in Fig. 3(b) indicated the corresponding grain boundaries in the AFM image, Fig. 3(a). The MFM image, Fig. 3(b), clearly showed a bright-dark contrast on each grain. Here, a simplified picture was proposed in Fig. 5(a). It presented the magnetic stray field of Fe films with magnetization direction pointed from right to left. Because the phase shift in MFM images is proportional to the magnetic force
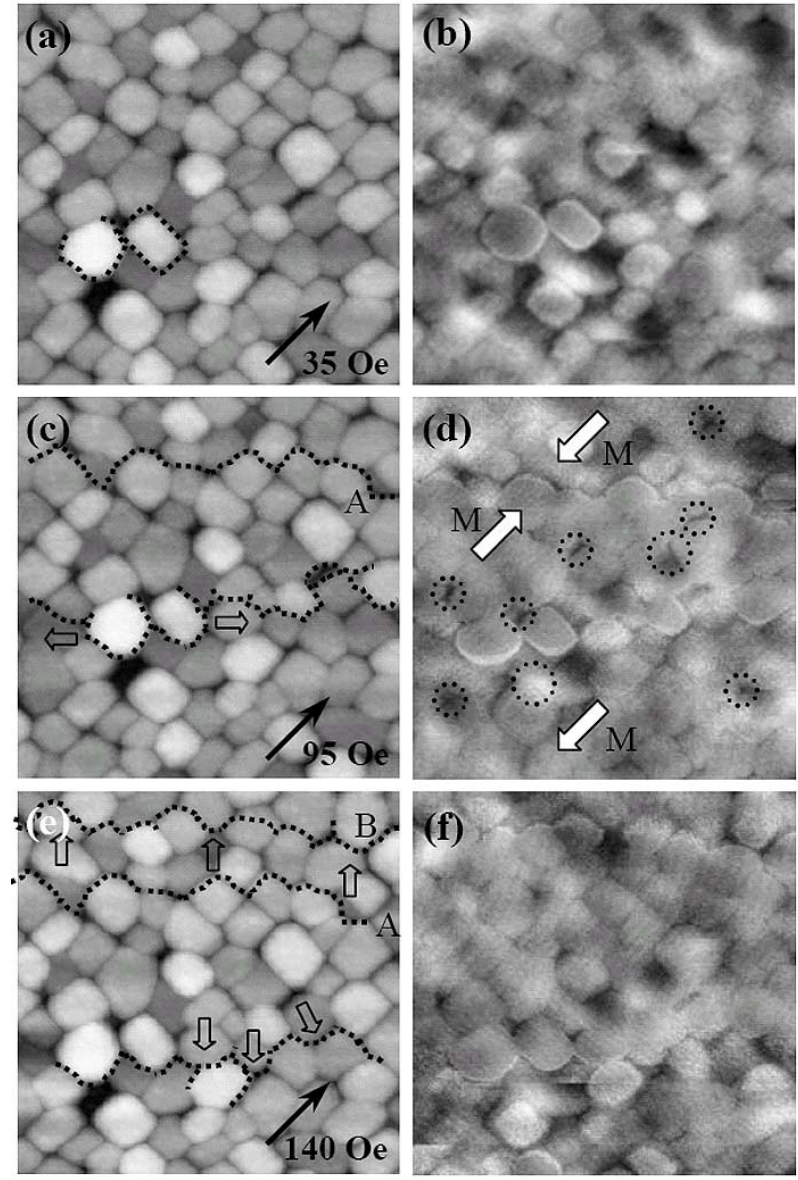

Fig. 4. A series of the $2 \times 2 \mu \mathrm{m}$ AFM (a), (c), and (e) and MFM (b), (d), and (f) images of Fe films under different external magnetic fields. Images (a)-(b), (c)-(d), and (e)-(f), were taken under 35, 95, and 140 Oe external magnetic fields, respectively, with a direction parallel to $\mathrm{Ag}[0-1-1]$ as indicated by the black arrows in (a), (c), and (e). (a)

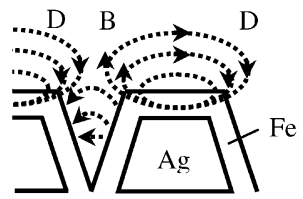

(c)

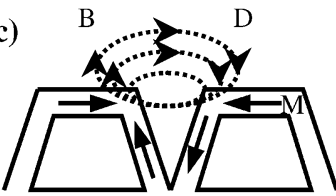

(b)
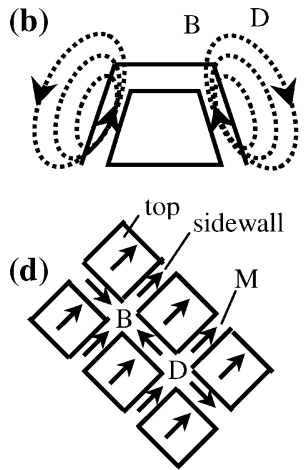

Fig. 5. Cartoon pictures for the distribution of the stray field (dotted lines) and magnetization state (solid arrows) of Fe films on $\mathrm{Ag}$ pyramidal islands. The alphabets " $B$ " and " $D$ " represent the bright and dark contrast in MFM image, respectively. Note that the magnetization direction of MFM tips is pointed downward.

gradient acts on the cantilever in the vibration direction. Therefore, the bright-dark contrast on each grain was caused by the single domain behavior of Fe films on each Ag island.

Fig. 4 shows a series of the AFM and MFM images of Fe films at the same position under different magnetic field strength with the direction parallel to $\mathrm{Ag}[0-1-1]$ direction. Note that Fig. 4 was taken after Fig. 3. That is the sample was first saturated 
along $\operatorname{Ag}[011]$ direction and then suffered a reverse field that was pointed to $\mathrm{Ag}[0-1-1]$ direction, the opposite direction to [011]. Fig. 4(b) showed the magnetic image of Fe films under field strength of $35 \mathrm{Oe}$. It displayed a complex and unrecognized domain pattern. Furthermore, the morphology signal seemed to couple into the MFM image. However, a peculiar domain pattern, as indicated by the corresponding dotted lines in Fig. 4(a), was observed in this image. It showed bright contrast that just located on the sidewalls of these two pyramidal islands. Due to shape anisotropy, the magnetization of Fe films grown on the sidewalls of $\mathrm{Ag}$ pyramidal islands is easier reversing on the sidewall (111) plane than others. Therefore, the magnetization lay on the sidewalls can cant an angle of $54^{\circ}$ to the film plane and produce a distribution of stray field like Fig. 5(b) that create a bright-dark contrast on the sidewall of pyramidal islands.

There appeared two wall-like structures in the MFM image of Fe sample, Fig. 4(d), under a magnetic field of 95 Oe. Each one of them composed of a set of bright-dark contrast. The corresponding dotted lines drawn in Fig. 4(c) showed that the position of wall-like structures was right on the sidewalls of pyramidal islands. By comparing Fig. 4(a)-(b) to (c)-(d), we found that the lower one of wall-like structures generated from the two islands that were first possessed canted magnetization as shown in Figs. 4(a) and (b). And this structure expanded along the direction indicated by the hollow arrows in Fig. 4(c). If we assumed that these special contrasts were magnetic domain walls and the magnetization inside domains has its direction as the white arrows shown in Fig. 4(d), then, a simple domain-wall model can be depicted in Fig. 5(c). Fig. 5(c) shows two magnetic domains (one points to right and the other goes left) met at the sidewalls of pyramidal islands. Because the adjacent sidewalls have different magnetization directions (one cants up and the other goes down), the magnetic stray field can be presented as Fig. 5(c). This type of domain structure propagated through neighboring islands and formed wall-like structures as observed in Fig. 4(d). As shown in Fig. 4(e) and (f), under a higher magnetic field, namely 140 Oe, the upper wall-like structure seemed to move from line "A" to line "B." And the lower one was also moved as indicated by the three hollow arrows in Fig. 4(e).

By comparing Fig. 4(c) and (d), at the joint of four islands, there was another interesting feature (circled by dotted rings in Fig. 4(d)) that displayed a bright or dark contrast in the MFM image. As depicted in Fig. 5(d), we assumed that the net magnetization inside a domain was pointed to up-right direction. However, the magnetization lay on the sidewalls that perpendicular to the direction of net magnetization needs a stronger external field to make them against the shape anisotropy. Therefore, in a low field state, namely $95 \mathrm{Oe}$, two possible configurations of magnetization can be sketched as Fig. 5(d), a bird's-eye view of the pyramidal islands. For the magnetization in a convergent (/diver- gent) state, the force gradient is positive (/negative) and a bright (/dark) contrast was shown in the MFM image. If a stronger external field, namely $140 \mathrm{Oe}$, was applied to the sample some features that circled in Fig. 4(e) were less contrast or disappeared in Fig. 4(f). In this study, we found that the magnetization lay on the sidewalls and the strength of external field were two important factors on the magnetization reversal of $\mathrm{Fe}$ films grown on $\mathrm{Ag}$ pyramidal islands. It stands to reason that $\mathrm{Fe}$ films possessed a perpendicular hysteresis behavior, because of the canted magnetization of Fe films grown on sidewalls of pyramidal islands.

\section{SUMMARY}

The Fe films grown on $\mathrm{Ag}$ submicrometer pyramidal islands with a (100) truncated top and four (111) sidewalls were successfully fabricated on $\mathrm{Si}(100)$ substrates through MBE technique and the magnetization reversal was studied for the first time. The magnetization reversal of Fe films first started at the sidewalls of pyramidal islands and followed by the formation and propagation of wall-like structures. The results indicated that the Fe films grown on the sidewalls of pyramidal islands and the strength of external field played important roles on the reversal phenomena.

\section{ACKNOWLEDGMENT}

This work was supported by the Academia Sinica and the National Science Council, Taiwan, R.O.C.

\section{REFERENCES}

[1] J. C. A. Bland and B. Heinrich, Ultrathin Magnetic Structures I and II. New York: Springer, 1994.

[2] A. Khapikov, L. Uspenskaya, J. Ebothe, and S. Vilain, Phys. Rev. B, vol. 57, p. $14990,1998$.

[3] J.-V. Kim, M. Demand, M. Hehn, K. Ounadjela, and R. L. Stamps, Phys. Rev. B, vol. 62, p. 6467, 2000.

[4] W. Weber, C. H. Back, A. Bischof, C. Wursch, and R. Allenspach, Phys. Rev. Lett., vol. 76, p. 1940, 1996.

[5] L. Gavioli, K. R. Kimberlin, M. C. Tringides, J. F. Wendelken, and Z. Zhang, Phys. Rev. Lett., vol. 76, p. 1940, 1996.

[6] W. S. Cho, J. Y. Kim, N. G. Park, K. H. Chae, Y. W. Kim, I. W. Lyo, S. S. Kim, D. S. Choi, and C. N. Whang, Surf. Sci., vol. 439, pp. L792-L798, 1999.

[7] J. Swerts, K. Temst, N. Vandamme, C. VanHaesendonck, and Y. Bruynseraede, J. Magn. Magn. Mater, vol. 240, pp. 380-382, 2002.

[8] W. Kern and D. A. Puotinen, RCA Rev., vol. 31, p. 187, 1970.

[9] M. Bollani, L. Fares, A. Charaï, and D. Narducci, Mater. Sci. Eng. B, vol. 73, p. 154, 2000

[10] C. C. Yu, W. C. Cheng, W. B. Lee, S. Y. Chen, Y. Liou, and Y. D. Yao, J. Appl. Phys., vol. 93, p. 7468, 2003.

[11] G. A. Prinz, "Magnetic metal films on semiconductor substrates," in Ultrathin Magnetic Structures, B. Heinrich and J. A. C. Bland, Eds. Berlin, Germany: Springer-Verlag, 1994, vol. II, pp. 1-5.

Manuscript received February 7, 2005 